\title{
Cascade Effects on the
} Polarization of He-like Fe 1s 2l $-1 s^{2} X$-ray Line Emission

P. Hakel, R. Mancini, C. Harris, P. Neill, P. Beiersdorfer, G. Csanak, and H. Zhang

January 10, 2007

Physical Review A 
This document was prepared as an account of work sponsored by an agency of the United States Government. Neither the United States Government nor the University of California nor any of their employees, makes any warranty, express or implied, or assumes any legal liability or responsibility for the accuracy, completeness, or usefulness of any information, apparatus, product, or process disclosed, or represents that its use would not infringe privately owned rights. Reference herein to any specific commercial product, process, or service by trade name, trademark, manufacturer, or otherwise, does not necessarily constitute or imply its endorsement, recommendation, or favoring by the United States Government or the University of California. The views and opinions of authors expressed herein do not necessarily state or reflect those of the United States Government or the University of California, and shall not be used for advertising or product endorsement purposes. 


\title{
Cascade effects on the polarization of He-like Fe 1s $2 \mathrm{l}-1 \mathrm{~s}^{2} \mathrm{X}$-ray line emission
}

\author{
Peter Hakel $\uparrow$, Roberto C. Mancini $\dagger$, Clifford Harris $\dagger^{1}$, Paul Neill $\uparrow$, Peter Beiersdorfer $\dagger$, \\ George Csanak§†, and Hong Lin Zhang§ \\ $\dagger$ Department of Physics, University of Nevada, Reno, NV 89557, USA \\ † Lawrence Livermore National Laboratory, Livermore, CA 94550, USA \\ $\S$ Los Alamos National Laboratory, Los Alamos, NM 87545, USA
}

\begin{abstract}
We calculate X-ray line polarization degrees for cases with axial symmetry using a collisional-radiative magnetic-sublevel atomic kinetics model and the properties of multipole radiation fields. This approach is well-suited for problems where the alignment is determined by the competition between many atomic processes. We benchmark this method against polarization measurements performed at the Livermore electron beam ion trap, and we study the 3-to-2 cascade effects on the polarization of 2-to-1 lines in He-like Fe.
\end{abstract}

PACS: 34.80.Dp, 34.80.Lx, 32.70.Cs, 32.80.Dz, 52.25.Os, 31.10.+z, 52.70.La, 52.38.Ph

\section{Introduction}

Polarized line emissions have been recognized as a signature of anisotropy in distributions of plasma electrons [1]. In particular, beams of plasma electrons can excite upper levels of line transitions in a non-statistical way, i.e., causing different magnetic sublevels of fine structure levels to have different populations. This condition called alignment reveals itself in partial polarization of emitted radiation. Beams of electrons occur in plasmas in several cases; electrons spiral along magnetic fields lines in solar corona [2,3] and electron beam ion trap (EBIT) experiments [4], in laser-produced plasmas electron beams are generated via nonlinear absorption of laser radiation [5-7]. Understanding the effects of the presence of directional electrons in plasmas is of fundamental importance. Frequently these electrons are very energetic compared to the thermal plasma electrons and excluding them from theoretical models may lead to serious overestimates of plasma temperature [8]. Also, the fast-igniter approach to inertial confinement fusion relies on a beam of fast electrons that can initiate a fusion burn. It is therefore desirable to develop diagnostic tools capable of characterization of non-thermal electrons' distribution properties in plasmas. Analyses of polarized line radiation from plasmas provide a way for doing that. Polarization-based diagnostics takes advantage of a degree of freedom of electromagnetic radiation not typically used in plasma spectroscopy in the past. Fundamental quantum mechanical studies are necessary in order to provide insights into the mechanism and

${ }^{1}$ present address: Gulf Coast Community College, Panama City, FL 32401, USA 
characteristics of polarized line emissions. Most of this work was done using densitymatrix formalism [9-13].

While polarization-based plasma diagnostics breaks new ground, good quality modeling for its purposes must face the same issues addressed by the more traditional radiationbased diagnostic techniques. Plasma spectroscopy has been a valuable tool for the determination of plasma characteristics in celestial as well as laboratory plasmas. Observed spectral line intensity ratios are yardsticks for measuring plasma temperature and charge balance; Stark-effect-induced broadening of line profiles contains information about plasma density. Theoretical reproduction of recorded spectra requires calculation of populations of the plasma ion species that are in their ground as well as excited states. In non-LTE plasmas, the populations often strongly deviate from the Boltzmann/Sahaequilibrium values. In order to address this issue multi-level collisional-radiative atomic kinetic models are constructed. Energy level populations are then calculated as a result of combined effects of many atomic processes. Many energy levels may need to be included in a model for satisfactory reproduction of experimental data, which in turn leads to large sizes of atomic databases. The size and complexity of a particular atomic kinetic model is determined by the level of detail used in the description of energy level structure. Since polarized radiation emerges from collections of ions with unequal populations of magnetic sublevels within individual fine-structure levels, development of fundamental, magneticsublevel atomic kinetic models is warranted. Such models must be complemented with a way of calculating polarized line emissions based on magnetic sublevel populations. Work in this direction has been done using density-matrix formalism [10]. We present another approach based on properties of multipole radiation fields, which agrees with the work of Inal and Dubau and is consistent with results of another density matrix method [11].

In this paper we report the results of the application of our technique to several experiments performed at the Livermore EBIT-II electron beam ion trap. Experimental conditions in EBIT are under very precise control, which makes EBIT very well suited for fundamental studies and tests. To this end we have constructed sublevel kinetics models (without cascades) for He-like $\mathrm{Si}$, and $\mathrm{Be}$ - and $\mathrm{B}$-like $\mathrm{Fe}$ and calculated polarizations of dielectronic satellite line emissions from these ions driven by monoenergetic electron beams. Having tested our method in these cases we then proceed to report on our calculations of polarized K-shell emissions from He-like Fe based on a sublevel kinetics model that includes cascade effects. Elsewhere we describe the results of the same procedure applied to a more complex plasma environment aimed at studying the polarization properties of Ly- $\square$ satellites in laser-produced Si plasma [14].

\section{Theory}


Previous calculations of polarized line emissions have used various implementations of density-matrix theory [9-13]. Here, we present a method for the calculation of polarized line emissions that is based on a collisional-radiative atomic kinetics model of magnetic sublevel populations. This technique is well suited for cases with cascade effects because it eliminates the need of a priori identification of dominant atomic processes. This method is also particularly useful for application in transient plasmas where different atomic processes may become important at different times. Magnetic sublevels are quantum states characterized by parity $\square$, energy $\mathrm{E}$, total angular momentum $\mathrm{J}$, and its projection $\mathrm{M}_{\mathrm{J}}$. In the absence of hyperfine interaction and external fields these are good quantum numbers. These quantum numbers are complemented by the dominant configuration and LS labels. We consider a fundamental collisional-radiative atomic kinetics model for calculating magnetic sublevel populations and, subsequently, polarization-dependent line spectra. This is accomplished by setting up a system of kinetic rate equations for the magnetic sublevels which, in general, is time-dependent. However, for the cases considered here we can assume steady-state conditions, and therefore the system of rate equations can be written down in matrix notation as follows,

$\underline{\underline{A g}}=\vec{b}$

where $\vec{g}$ is the vector of magnetic sublevel populations, $\underline{\underline{A}}$ is the rate matrix associated with the atomic processes included in the model, and $\vec{b}$ is a vector that depends on populations not computed by the model and thus considered to be input (e.g., ground state populations in the applications discussed here). The number of magnetic sublevels in the model determines the number of rate equations in the system, and the number and type of atomic processes included determines the level of coupling between the equations. Implicit in the model is the selection of an axis of quantization with respect to which the magnetic sublevel quantum number $M$ and the direction of observation are referred to, and axial symmetry is assumed; we take the z-coordinate axis to be this axis.

In general, to address the problem of calculating polarized line emission one has to consider the density matrix of the ion and electromagnetic field system. Then, the photon density matrix can be extracted which contains the Stokes parameters that characterize the polarization properties of the radiation field. The diagonal elements of the ion density matrix are the magnetic sublevel populations, and the off-diagonal elements represent coherences. However, for the case of systems with axial symmetry the ion-density matrix remains diagonal and the line polarization properties are only characterized by magnetic sublevel populations [10]. Hence, a line transition is formed as an incoherent collection of sublevel transitions that are inherently polarized due to angular momentum conservation of the ion and photon system. Pure multipole radiation emissions from optically thin sources due to transitions between magnetic sublevels have distinct angular and polarization characteristics [15]. It is customary to observe polarized emissions in a direction perpendicular to the chosen quantization axis. In the case of an electric dipole 
(E1) line whose upper and lower states have the same value of the magnetic quantum number $\mathrm{M}_{\mathrm{J}}$, the emission is linearly polarized parallel to the axis of quantization. On the other hand, if the magnetic quantum number changes by one unit, an E1 emission is polarized in a direction perpendicular to the quantization axis. The polarization of emissions from sublevel-to-sublevel transitions is determined by the multipole type of the transition and the absolute difference in $\mathrm{M}_{\mathrm{J}}$ values of the upper and lower levels. This is a manifestation of angular momentum conservation of the ion + radiation field system during the ion's decay by photon emission. A fine structure line $\mathrm{J}_{\mathrm{i}} \square \mathrm{J}_{\mathrm{f}}$ consists of sublevel lines polarized in both directions, which overlap due to degeneracy with respect to $\mathrm{M}_{\mathrm{J}}$. Hence, in the optically thin approximation, we calculate polarization-dependent fine-structure line intensities by,

$$
I_{\|, \square} \quad h \square A\left(J_{i} \square J_{f}\right) \square \prod_{M_{i}=\square J_{i}}^{J_{i}} f\left(M_{i}\right) \prod_{M_{f}=\square J_{f}}^{J_{f}} M I_{\|, \square}\left(\square M, \square=90^{\circ}\right) \square\left(\begin{array}{llllll}
J_{f} & q & M_{f} \quad \square \square M & J_{i} & \left.M_{i}\right)^{2}
\end{array}\right.
$$

where $\mathrm{h} \square$ is the transition energy, $\mathrm{A}\left(\mathrm{J}_{\mathrm{i}} \square \mathrm{J}_{\mathrm{f}}\right)$ is the transition's radiative decay rate, $\mathrm{f}\left(\mathrm{M}_{\mathrm{i}}\right)$ are populations of upper level's sublevels, $\square$ is the angle between the quantization axis and the line of sight, $q$ is the multipolarity of the transition, $\square \mathrm{M}=\mathrm{M}_{\mathrm{f}}-\mathrm{M}_{\mathrm{i}}$, and $\left(\begin{array}{llll}J_{f} & q & M_{f} & \square \square\end{array} \mid \begin{array}{ll}J_{i} & M_{i}\end{array}\right)$ is a Clebsch-Gordan coefficient. The products of Clebsch-Gordan coefficients and $\mathrm{A}\left(\mathrm{J}_{\mathrm{i}} \square \mathrm{J}_{\mathrm{f}}\right)$ are the spontaneous radiative decay rates of the constituent magnetic sublevel transitions [16]. $\mathrm{MI}_{-}(\square \mathrm{M}, \square)$ 's are relative multipole intensities based on wave-zone multipole fields, in particular their angular parts are also known as vector spherical harmonics [15], and $\square$ is the angle between the direction of observation and the axis of symmetry (same as the polar angle). Their values for dipole and quadrupole transitions are listed in Tables I and II. The two polarization-dependent line intensities observed at $\square=90^{\circ}$ then yield the polarization degree,

$$
P=\frac{I_{\|} \square I_{\square}}{I_{\|}+I_{\square}}
$$

Under isotropic conditions (LTE plasmas, for instance) populations of magnetic sublevels within a fine-structure level are the same, which results in unpolarized line emissions. Polarization may therefore arise only from lines whose upper levels are aligned, i.e., the population is unequally distributed among their magnetic sublevels. Alignment can be created by anisotropic processes such as electron collisional excitation or electron capture driven by electron beams.

Here, all atomic structure, rate and cross section data is calculated with the Los Alamos atomic structure and scattering codes CATS, ACE, and GIPPER. CATS is a multiconfiguration Hartree-Fock atomic structure code that computes energy level 
structure characteristics (eigenstates' wave functions and energies) and transition matrix elements for spontaneous radiative decay rates [17]. The electron scattering code ACE uses atomic structure data computed by CATS to calculate electron-impact excitation scattering amplitudes and cross sections in the Born, Coulomb, or distorted wave approximation for transitions between magnetic sublevels, fine-structure levels, LS-terms and configuration-averaged states [18]. In its sublevel mode ACE provides differential cross sections which we integrated over the scattering angle. The ionization code GIPPER also uses atomic structure data from CATS to calculate autoionization rates and cross sections for electron-impact ionization and photoionization [19]. This suite of codes can efficiently generate large databases for atomic kinetics modeling.

\section{Polarization of dielectronic satellite lines}

In this section we discuss the results of our calculations for the polarization degrees of dielectronic satellite lines in Fe ions, and compared them with measurements at the Livermore EBIT as well as independent calculations [20-22]. In particular, we consider 1s $212 l^{\prime} 2 l^{\prime \prime} \square 1 s^{2} 212 l^{\prime}$ transitions in Be-like Fe and 1s $212 l^{\prime} 2 l^{\prime \prime} 2 l^{\prime \prime} \square 1 s^{2} 212 l^{\prime} 2 l^{\prime \prime}$ in B-like Fe where $l, l^{\prime}, l^{\prime \prime}$ and $l^{\prime \prime \prime}$ can be $s$ or $p$. An electron beam is capable of creating alignment in the upper (autoionizing) levels of dielectronic satellite lines by populating them via resonant electron capture. The potential anisotropy of this process stems from the conservation of the z-component of the total angular momentum of the system. The continuum electron has $m_{l}=0$ which leads to the selection rule $|\square \mathrm{M}|=1 / 2$ between the two ionic states involved in the electron capture process [23]. For the low density conditions at EBIT $\left(\mathrm{N}_{\mathrm{e}} \square 1 \square 10^{12} \mathrm{~cm}^{-3}\right)$ we can assume that each autoionizing state is populated solely by electron capture from the ground state of the next higher ionization stage, and depopulated by autoionization and spontaneous radiative decays. Furthermore, the energy of the electron beam can be tuned in such a way that only subsets of electron capture resonances are excited. Hence, the populations of autoionizing magnetic sublevels can be calculated from a set of de-coupled kinetic rate equations (see Eq. 1) given the rates of electron capture, autoionization and spontaneous radiative decays, and the population of the ground state of the next higher ionization stage. Actually, we have normalized this ground state population to 1 so that all other populations in our model are calculated in units of the ground state population. Sublevel radiative decay rates for pure multipole radiative transitions are calculated from the J-level rates by the following formula which is based on the Wigner-Eckart theorem [16],

$$
A\left(J_{i} M_{i} \square J_{f} M_{f}\right)=A\left(J_{i} \square J_{f}\right) \square\left(\begin{array}{llllll}
J_{f} & q & M_{f} & \square \square M & \mid J_{i} & M_{i}
\end{array}\right)^{2}
$$

While this is a general relationship, magnetic sublevel autoionizing and electron capture rates can also be written in terms of the corresponding J-level rates under the approximation that this process occurs through a single dominant open channel. A detailed discussion of the calculation of these rates is given in the Appendix. We note that each individual magnetic sublevel JM of a given level $\mathrm{J}$ decays with the same total 
radiative (as seen from summing over $\mathrm{M}_{\mathrm{f}}$ in Eq. 4) and autoionization decay rates (Eq. A12), respectively. This reflects the fact that these processes are isotropic.

Spontaneous radiative decay and autoionization rates for fine structure J-levels were computed with the codes CATS and GIPPER, and then used to compute magnetic sublevel JM rates according to Eq. (4) and the method discussed in the Appendix. Next, magnetic sublevel populations and polarization degrees were computed according to the model discussed in the theory section. Our results agree with the B- and Be-like Fe measurements at EBIT, and also with results from independent calculations based on the photon density matrix [20,22]. The only exception is line \#9 in Table I of Ref. [20] (1s 2s $\left.2 \mathrm{p}^{2}\{1\}^{3} \mathrm{P}_{2} \square 1 \mathrm{~s}^{2} 2 \mathrm{~s} 2 \mathrm{p}^{3} \mathrm{P}_{2}\right)$ in Be-like Fe. This line has not one but two open dominant transition channels from terms ${ }^{1} \mathrm{D}$ and ${ }^{3} \mathrm{D}$ and therefore our single-channel assumptions about its electron capture rates do not apply. Even for this line, however, we reproduce the two limiting values of -1 and $-3 / 7$ depending on which LS-term is taken as dominant, also in accordance with Ref. [20]. Also, polarization degree results for the He-like $\mathrm{Si}$ satellites of the Ly- $\square$ agree with those of Ref. [21].

\section{Cascade effects on the polarization of lines $w, x, y$, and $z$ in He-like Fe}

Beiersdorfer et al. measured the polarization of the $1 \mathrm{~s} 2 \mathrm{p}{ }^{1} \mathrm{P}_{1} \square 1 \mathrm{~s}^{2}{ }^{1} \mathrm{~S}_{0}$ resonance line $w$ (E1), $1 \mathrm{~s} \quad 2 \mathrm{p}{ }^{3} \mathrm{P}_{2} \square \quad 1 \mathrm{~s}^{2}{ }^{1} \mathrm{~S}_{0}$ intercombination line $x$ (M2), $1 \mathrm{~s} \quad 2 \mathrm{p} \quad{ }^{3} \mathrm{P}_{1} \quad \square \quad 1 \mathrm{~s}^{2}{ }^{1} \mathrm{~S}_{0}$ intercombination line $y(\mathrm{E} 1)$, and $1 \mathrm{~s} 2 \mathrm{~s}^{3} \mathrm{~S}_{1} \square 1 \mathrm{~s}^{2}{ }^{1} \mathrm{~S}_{0}$ forbidden line $z$ (M1) from He-like Fe ions in EBIT experiments [11]. These emissions were driven by an unpolarized, monoenergetic (6.8 keV) electron beam. Using Eqs. of (2) and (3) we can express the polarization of these lines in terms of upper level magnetic sublevel populations $f\left(M_{i}\right)$ as,

lines $w$ and $y$ :

$$
P=\square \frac{f(\square 1) \square 2 f(0)+f(+1)}{f(\square 1)+2 f(0)+f(+1)}
$$

line $x$ :

$$
P=\frac{f(\square 2) \square f(\square 1) \square f(+1)+f(+2)}{f(\square 2)+f(\square 1)+f(\square 1)+f(+2)}
$$

line $z$ :

$$
P=\frac{f(\square 1) \square 2 f(0)+f(+1)}{f(\square 1)+2 f(0)+f(+1)}
$$


which are consistent with the formulae of Inal and Dubau [10]. Under the steady-state conditions of an EBIT and the assumption of no cascades, the upper sublevels of these transitions are populated solely by direct electron-impact excitation from the ground state characterized by sublevel cross sections $\square_{\mathrm{M}}$. Since radiative decay is isotropic, we have $\mathrm{f}(\mathrm{M}) \quad \square_{\mathrm{M}}$ and the polarization formulae (5)-(7) agree with that of Ref. [11] with the cross sections in place of sublevel populations. This approximation only holds for such special cases, and these "2-level-atom" polarization results containing sublevel cross sections have to be modified in the presence of other feeding channels for the upper level. Such effects are automatically accounted for in our multilevel atomic kinetic models, which yield sublevel populations, and therefore require no formal modification of expressions (5)-(7).

Our atomic kinetic model for He-like Fe consists of a total of 53 magnetic sublevels from configurations $1 \mathrm{~s}^{2}, 1 \mathrm{~s} 2 \mathrm{l}$, and $1 \mathrm{~s} 31$. In order to address the effects of $1 \mathrm{~s} 31 \mathrm{l}$, 21 radiative decay cascades we calculated polarization of the four lines at electron beam energies 6.8 and $8.0 \mathrm{keV}$. Due to the small energy dispersion of EBIT electron beams $(\square 50 \mathrm{eV})$ the electrons do not excite the 1s 3131 ' resonances that lie in the $6.95-7.01 \mathrm{keV}$ range (see Fig. 1) and whose effects to $1 \mathrm{~s} 21$ excitation have been studied by Inal and Dubau [23]. The 1s 31 excitation threshold is $7.9 \mathrm{keV}$, hence at $6.8 \mathrm{keV}$ the $1 \mathrm{~s} 31$ states acquire only negligible populations by stepwise excitation via $1 \mathrm{~s} 21$ states. Therefore only cascades within the $1 \mathrm{~s} 21$ states may play a role at $6.8 \mathrm{keV}$. At $8.0 \mathrm{keV}$ the $1 \mathrm{~s} 31$ states become accessible to direct excitation from the ground state which turns on the $\mathrm{n}=3$ to $\mathrm{n}=2$ cascades. It is straightforward to exclude any levels from the model and thus identify dominant feeding channels by looking at the sensitivity of the final result. Considering the low electron density of the beam $\left(10^{12} \mathrm{~cm}^{-3}\right)$ it is sufficient to include only electronimpact excitation and spontaneous radiative decay processes. Using the suite of Los Alamos codes we constructed a database of 50 rates of electric dipole transitions between J-levels. Our model also includes the rates of three higher-order transitions, namely those associated with lines $x$ and $z$ [24] and the two-photon decay $1 \mathrm{~s} 2 \mathrm{~s}{ }^{1} \mathrm{~S}_{0}{ }_{1}{ }^{1 \mathrm{~s}^{2}}{ }^{1} \mathrm{~S}_{0}$ [25]. Inclusion of these processes is necessary because upper levels of these three transitions lack dominant electric dipole decay channels

Electron-impact excitations are represented by a database of 1296 sublevel excitation cross sections created with the Los Alamos code ACE. (There is some redundancy in the database because sublevel cross sections are the same if $M_{J}$ values of the initial and final states both change sign.) Since the results of kinetic models depend on the quality of the employed atomic database, we also obtained another collection of cross sections independently calculated by a fully relativistic code [26]. This allows us to investigate the sensitivity of our results with respect to various collections of atomic data produced under different methods and approximations. The comparisons between these two 
collections of data representing excitations of the upper levels of lines $w, x, y$, and $\mathrm{z}$ from the ground state (Table III) show that the relativistic corrections play a small role, as is expected for a light element such as Fe. While the excitation data of $1 \mathrm{~s} 2 \mathrm{~s}{ }^{3} \mathrm{~S}_{1}$ level show the largest differences between the two calculations, the relative values of individual sublevel cross sections remain virtually unchanged (see Figs. 2 and 3) even for this level. Since the degree of polarization is determined by the relative difference of upper level's sublevel populations, the "2-level" polarization results are essentially the same regardless of which cross section database is used in the model.

Our results are summarized in Tables IV and V. The columns labeled "17-level" represent the results obtained from our full model, while the "2-level" results refer to separate calculations involving only the two levels of each transition. The comparison of the "2level" results obtained from the two databases reflects the changes in the relative magnitudes of the direct-excitation sublevel cross sections. These differences between the two databases are illustrated in the form sublevel cross section ratios in Figs. 2 and 3. For example, the excitation of the $1 \mathrm{~s} 2 \mathrm{~s}^{3} \mathrm{~S}_{1}$ level is isotropic in the ACE model while the relativistic ("Zhang") calculations predict a tiny alignment creation in this process. This translates into the -0.0051 at $6.8 \mathrm{keV}$ and -0.0055 at $8.0 \mathrm{keV}$ "2-level" polarization result for line $z$ as opposed to the exact 0 in the ACE column. The line $w$ has a positive degree of polarization because the $\mathrm{M}=0$ sublevel of the upper ( $\left.1 \mathrm{~s} 2 \mathrm{p}{ }^{1} \mathrm{P}_{1}\right)$ level has a larger cross section than the $M= \pm 1$ sublevels (see Eq. 6). This difference in sublevel cross sections is more pronounced in the ACE data which leads to even more positive "2-level" polarization result than that based on the fully relativistic ("Zhang") data.

It is the difference between the "2-level" and the corresponding "17-level" results that is attributable to cascades. At $6.8 \mathrm{keV}$ the only line whose polarization is noticeably altered by cascades is the line $z$. Direct excitation of the $1 \mathrm{~s} 2 \mathrm{~s}{ }^{3} \mathrm{~S}_{1}$ level from the ground state is essentially isotropic as is apparent from the vanishing "2-level" result. Its noticeable negative polarization is attributed to alignment transfer from the $1 \mathrm{~s} 2 \mathrm{p} \quad{ }^{3} \mathrm{P}_{2}$ state (polarization goes back to zero, if this level is excluded from the calculation). This state is aligned by anisotropic excitation from the ground state which manifests itself in the polarization of line $x$, for which $1 \mathrm{~s} 2 \mathrm{p}^{3} \mathrm{P}_{2}$ is the upper level. Our results are in agreement with experimental results of Beiersdorfer et al. (see [11] and Table IV).

For an electron energy of $8.0 \mathrm{keV}$ radiative cascades from $\mathrm{n}=3$ singly excited states turn on. Adding more energy levels to our model is not necessary because this energy is still below the $8.2 \mathrm{keV}$ threshold for the $\mathrm{n}=4$ singly excited states. Lines $w$ and $x$ do not undergo any major changes but lines $y$ and $z$ exhibit interesting properties. New measurements were performed at the Livermore EBIT-II electron beam ion trap at the beam energy of $8.0 \mathrm{keV}$ using similar techniques as those reported earlier [11]. The results are listed in Table $\mathrm{V}$. These measurements indicate that the polarization of line $x$ actually does change a bit, to the point that our calculated result falls out of the error bar. We should note, however, that polarization measurements are generally very challenging, 
and the error bars given are purely statistical. Unknown systematic effects, possibly due to inhomogeneous crystal reflectivities or inhomogeneous focusing properties and the like, may alter the results and are very difficult to detect. On the other hand, because of the inherent properties of the two-crystal measurement technique used, the uncertainties are intrinsically smallest for lines with the most negative polarization (and conversely largest for those with the most positive polarization). As a result, the measurement of the polarization of line $x$ carries the smallest uncertainty. Good agreement by contrast is found for lines $w, y$, and $z$, albeit near or right at the edge of the respective error bars.

Since the "2-level" polarization of line $z$ is remains zero at $8.0 \mathrm{keV}$, and a calculation at this energy with the $n=3$ states excluded yields a value around -0.08 , the significant increase of polarization up to -0.15 is caused by cascades from $1 \mathrm{~s} 31$. We pinpointed the most significant feeding channels of $1 \mathrm{~s} 2 \mathrm{~s}{ }^{3} \mathrm{~S}_{1}$ and constructed a 5-level model in which these effects on line $z$ can be illustrated. This five-energy-level subset is: $1 \mathrm{~s}^{2}{ }^{1} \mathrm{~S}_{0}, 1 \mathrm{~s} 2 \mathrm{~s}$ ${ }^{3} \mathrm{~S}_{1}, 1 \mathrm{~s} 2 \mathrm{p}{ }^{3} \mathrm{P}_{0}, 1 \mathrm{~s} 2 \mathrm{p}{ }^{3} \mathrm{P}_{2}$, and $1 \mathrm{~s} 3 \mathrm{p}^{3} \mathrm{P}_{2}$. Our results (see Table VI) show that the cascade from $1 \mathrm{~s} 3 \mathrm{p}^{3} \mathrm{P}_{2}$ further enhances the negative polarization value seen also at $6.8 \mathrm{keV}$ that was attributed to the cascade from $1 \mathrm{~s} 2 \mathrm{p}{ }^{3} \mathrm{P}_{2}$. The line emission associated with this cascade is itself noticeably polarized $(\mathrm{P}=+0.29)$ which demonstrates that alignment transfer from $1 \mathrm{~s} 3 \mathrm{p}^{3} \mathrm{P}_{2}$ to $1 \mathrm{~s} 2 \mathrm{~s}^{3} \mathrm{~S}_{1}$ is indeed taking place. On the other hand, these two cascades compete with the isotropic cascade from $1 \mathrm{~s} 2 \mathrm{p}{ }^{3} \mathrm{P}_{0}$ level. These findings are consistent with earlier calculations of Inal and Dubau [23], who accounted for cascades by calculating effective collision strengths for the $1 \mathrm{~s}^{2}{ }_{-} 1 \mathrm{~s} 21$ excitations at several energies, of which the one closest to our calculations is $7.9 \mathrm{keV}$ (582 Ry). Most importantly, this significant enhancement of the polarization of line $z$ at $8.0 \mathrm{keV}$ in comparison to the value at $6.8 \mathrm{keV}$ is also observed by our newly reported experimental results (see Table V). Fig. 4 shows the most dominant atomic processes in the kinetics of the upper level of line $z$. The "population $\square$ rate" values are the measure of influence of a particular atomic process on the population of the $1 \mathrm{~s} 2 \mathrm{~s}{ }^{3} \mathrm{~S}_{1}$ level. The fact that several feeding channels are of comparable importance and that the direct excitation from the ground state is not even the most dominant among them illustrates the critical importance of considering complex cascade effect patterns. Hereby presented magnetic-sublevel kinetic modeling addresses these issues by definition. This is an improvement with respect to previous efforts that start with a "2-level" type modeling and add cascades on a case-by-case basis.

The "2-level" result for line $y$ is considerably smaller at $8.0 \mathrm{keV}$ than the corresponding value at $6.8 \mathrm{keV}$. This is a consequence of sublevel excitation cross section $\square_{l}$ being much more comparable to $\square_{2}$ at $8.0 \mathrm{keV}$ than at $6.8 \mathrm{keV}$, making excitation from the ground state less anisotropic. Further reduction of polarization seen in the comparison of the "2-level" and "17-level" models at $8.0 \mathrm{keV}$ is then due to cascades; however, in this case there are no dominant population channels that would allow us to recover the "full" result with a small number of included levels. Therefore, the polarization (or, in this case, the lack thereof) of line $y$ at $8.0 \mathrm{keV}$ is indeed a product of complex multilevel sublevel atomic kinetics, which would otherwise be more difficult to calculate with more traditional ("2- 
level-based" with possible cascades) models. This theoretical result is also supported by the experimental observation on EBIT-II.

\section{Summary and conclusions}

We have developed an alternative approach to calculations of polarized line emissions. Our method is based on collisional-radiative atomic kinetic modeling of magnetic sublevel populations and line polarization is calculated using the properties of multipole radiation fields. We have benchmarked our results with EBIT experiments and independent calculations, and we have discussed in detail the 3-to-2 cascade effects on the 2-to-1 line transitions in He-like Fe. The highlight of this technique is its capability to include cascade and other multilevel effects in a straightforward manner. This makes our approach a good candidate for modeling of polarized line emissions from plasmas (laserproduced, for example) where there are many competing feeding channels whose relative importance will vary with changing plasma conditions. As an application of the technique described in this work, we have carried out a modeling study of polarization effects in Ly- $\square$ satellite lines in laser-produced Si plasma, which is presented in another publication [14].

\section{Appendix}

In this Appendix we derive the expressions for magnetic-sublevel autoionization rates. We also obtain the rate coefficients for magnetic-sublevel electron capture process driven by beam electrons. [27,28] We do this in the context of LS coupling [29].

\section{Autoionization}

We label the ionic states with the angular momentum quantum numbers $\mathrm{L}, \mathrm{S}, \mathrm{J}$, and $\mathrm{M}_{\mathrm{J}}$. Because of the spin-orbit interaction $\mathrm{L}$ and $\mathrm{S}$ are only approximate quantum numbers and are typically adopted as state labels from the dominant pure LS-coupled angular momentum eigenstate in the make-up of the ionic state. The rate of autoionization is given by the Fermi's Golden Rule,

$$
\text { a.i. rate } \left.=\frac{2 \square}{\hbar} \square_{f}\left(E_{f}\right) \mid\langle\text { initial }| H \square \text { final }\right\rangle\left.\right|^{2} \square\left(E_{f} \square E_{i}\right)
$$

where $H^{\prime}$ is the Hamiltonian for the electrostatic repulsion between the ion's electrons, $\square_{f}\left(E_{f}\right)$ is the density of final states, and the delta function represents the energy conservation requirement. $\mathrm{H}^{\prime}$ is a scalar operator and does not act on electron spin, which 
leads to the following properties and selection rules: (1) the initial and final states must be of the same parity, (2) the angular momentum quantum numbers $\mathrm{L}, \mathrm{S}, \mathrm{J}$, and $\mathrm{M}_{\mathrm{J}}$ must be the same for both states, and (3) the H' matrix element in (A1) is independent of J and $\mathrm{M}_{\mathrm{J}}$. [29]

The energy levels in an ion are mixtures of pure LS-coupled states. For the cases studied here the degree of mixing is small and thus in the following we represent the final ionic state by its dominant LS-coupled state $|L \$ \Phi M \square\rangle$. This state has to be further coupled with a one-electron continuum state in order to form the "final" state in Eq. (A1). The initial (autoionizing) state can be represented in a similar way by the $\left|\widetilde{L} \widetilde{S} J M_{J}\right\rangle$ LScoupled state. This state is selected as the one that satisfies the parity conservation requirement in the autoionization process [29]. Therefore this state is not always identical to the $\left|L S J M_{J}\right\rangle$ state that forms most of the initial autoionizing state. In the following we assume that there is a single dominant open channel associated with the orbital angular momentum value $\tilde{l}$ of the continuum electron and the (initial) LS-coupled state $\left|\widetilde{L} \widetilde{S} J M_{J}\right\rangle$ from which the largest part of the rate (A1) comes from. The possible values for $\tilde{l}$ are severely restricted by the simultaneous requirements of: (1) parity conservation, and (2) total angular momentum conservation with low final-state values of J' (typically 0 or _). Thus, our single-open-channel approximation is valid for almost all cases that we consider in this work. Hence, the derivation of the magnetic-sublevel formula only requires angular-momentum recoupling considerations. This approximation allows us to calculate sublevel rates from J-level rates (which we assume to be known) using the Clebsch-Gordan coefficients instead of calculating them "from scratch." Ultimately, this leads to the fact that the polarization degrees of the studied dielectronic satellite lines are rational numbers [20,22].

We introduce the following notation (only angular momentum quantum numbers are listed explicitly):

Initial (autoionizing) state: $\left|\widetilde{L} \widetilde{S} J M_{J}\right\rangle$

Final state (in the next higher ionization stage and the ejected electron):

“uncoupled" representation - $|L \Phi \square M \square\rangle\left|\widetilde{l} m_{l} m_{s}\right\rangle$

“coupled" representation - $\left|\widetilde{L} \widetilde{S} J M_{J}(L \$ \widetilde{\mathbb{Z}})\right\rangle$

The $\mathrm{L}, \mathrm{S}, \mathrm{J}$, and $\mathrm{M}_{\mathrm{J}}$ quantum numbers for the "coupled" final state are the same as those for the initial (autoionizing) state due to the selection rules in (A1) described above. The 
eigenstates in the "uncoupled" and "coupled" representations (A3) and (A4) are related by,

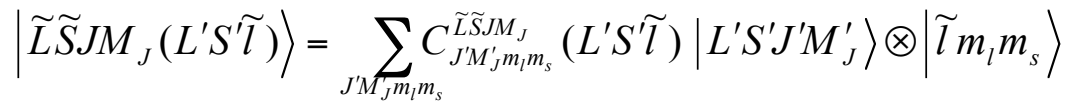

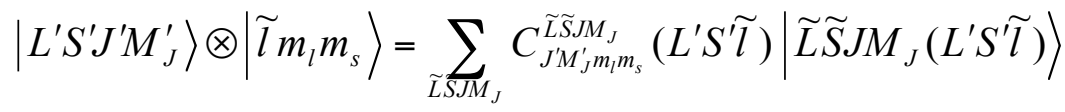

where,

$$
\begin{aligned}
& C_{J \llbracket I m_{l} m_{s}}^{\tilde{L} \widetilde{S} J M_{J}}(L \Phi \widetilde{\mathbb{Z}})=\bigsqcup_{M \square=\square L \square}^{L \square}(L \square S \square M \square \quad M \square \square M \square \mid J \square M \square) \square
\end{aligned}
$$

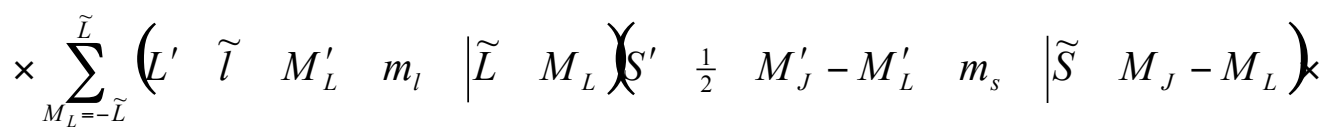

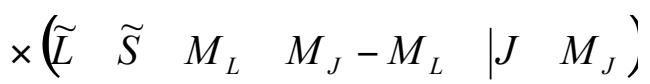

are the matrix elements of the unitary transformation operator associated with the change of angular momentum representation. These matrix elements are collections of ClebschGordan coefficients from which they inherit the unitarity property,

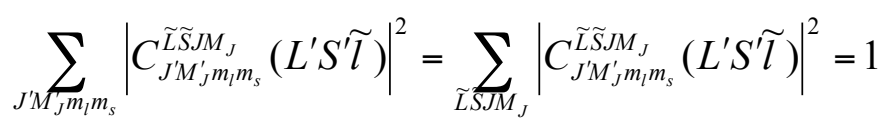

The sublevel-to-sublevel autoionization rate is given by,

$$
\left.A\left(L S J M_{J} \square L \$ \square M \square\right) \square_{m_{l}=\square \widetilde{l}}^{\tilde{l}} \square_{m_{s}= \pm \frac{1}{2}}\left|\left\langle\widetilde{L} \widetilde{S} J M_{J} \mid H \square L \$ \square M \square\right\rangle\right| \tilde{l} m_{l} m_{s}\right\rangle\left.\right|^{2}
$$

which is related to the rate $\mathrm{A}_{\mathrm{a}}$ defined by,

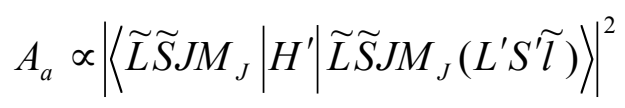

through the matrix elements defined in (A7). The rate (A10) is independent of $M_{J}$, hence,

$$
A_{a}=A\left(L S J M_{J} \square L \$ \square=A\left(L S J \square L \$ \square=\prod_{J \square} A(L S J \square L \$ \square \square\right.\right.
$$


The total sublevel depopulation rate due to autoionization is,

$$
A\left(L S J M_{J}\right)=\square_{L \$ \square \square} A\left(L S J \square L \$ \square \square=\bigsqcup_{L \rrbracket \square} A_{a}\right.
$$

which is isotropic (i.e., independent of $\mathrm{M}_{\mathrm{J}}$ ). Sublevel-to-sublevel rates are,

$$
A\left(L S J M_{J} \square L \$ \square M \square\right)=A_{a} \square F\left(\widetilde{L} \widetilde{S} J M_{J} \square L \$ \rrbracket M \square\right)
$$

where,

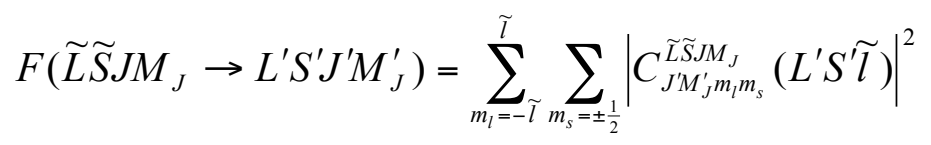

By defining,

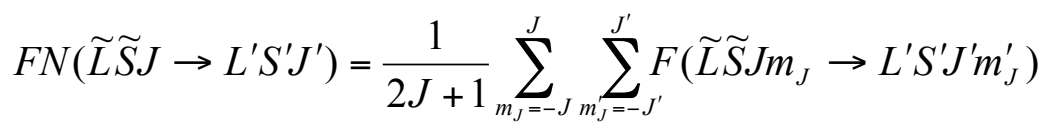

we can relate the sublevel and J-level autoionization rates as,

$$
A\left(L S J M_{J} \square L \$ \square M \square\right)=\frac{F\left(\tilde{L} \widetilde{S} J M_{J} \square L \$ \square M \square\right)}{F N(\widetilde{L} \widetilde{S} J L \$ \square \square} A(L S J \square L \$ \square \square
$$

\section{Electron capture driven by a unidirectional, unpolarized electron beam}

The cross section for the electron capture is given by an expression analogous to (A1) and (A9) with one important modification. In the study of the autoionization process the direction of propagation of the continuum electron was unimportant, while here the electron is moving along the $z$-axis. The $z$ component of its orbital angular momentum is zero $\square$ therefore, instead of summing over $m_{l}$, we set $m_{l}=0$ in (A9) and (A7). Also, we average over the continuum electron's two spin states. Thus we can define,

$$
\begin{aligned}
& G\left(L \$ \square M \square \widetilde{L} \widetilde{S} J M_{J}\right)=
\end{aligned}
$$

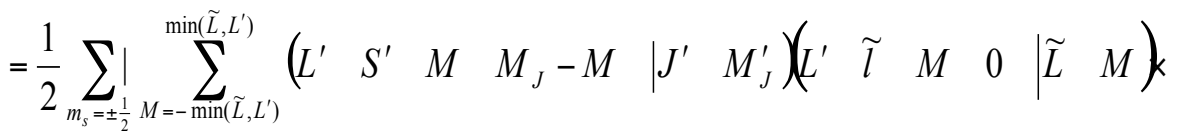




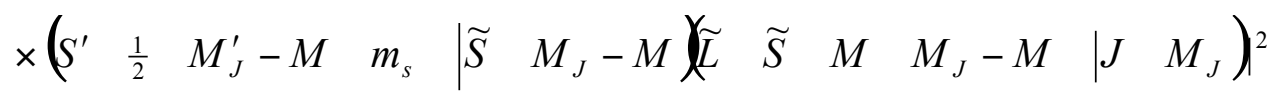

and,

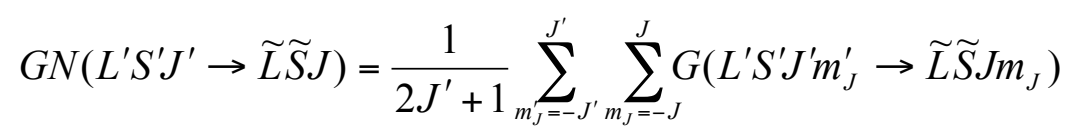

The sublevel and J-level electron capture cross sections are related by an expression analogous to (A16),

$$
\square\left(L \$ \square M \square \square L S J M_{J}\right)=\frac{G\left(L \$ \square М \square \square \widetilde{L} \widetilde{S} J M_{J}\right)}{G N(L \$ \square \square \square \widetilde{L} \widetilde{S} J)} \square(L \$ \square \square L S J)
$$

The sublevel electron capture rate coefficient is then given by $\langle v \square\rangle$, i.e., the average of the electron velocity $v$ and the electron capture cross section (A19) over the beam distribution function $f_{B}(E)$. The velocity and kinetic energy of the electron are related by,

$$
v=\sqrt{\frac{2 E}{m}}
$$

where $m$ is electron mass.

The electron capture is a resonant process. Thus, if we denote the kinetic energy of the free electron needed for the capture as $E_{r}$, we can write [30],

$$
\square(E)=B \square\left(E \square E_{r}\right)
$$

where $\square(E)$ represents the dependence of the J-level cross section from the right-hand side of Equation (A19) on electron energy $E$. Like all J-level atomic data, this cross section is isotropic, i.e., it is independent of the direction of the electron beam. Therefore, the magnitude factor $B$ in (A21) can be obtained by evaluating the rate coefficient integral for a normalized Maxwellian electron distribution function $f_{\operatorname{Maxw}}(v, T)$,

$$
\left.f_{\text {Maxw }}(v, T)=4 \square \frac{m}{2 \square k T}\right]^{\frac{3}{2}} v^{2} e^{\frac{m v^{2}}{2 k T}}
$$


and comparing it with the expression based on the detailed-balance principle (see below).

Thus for the rate coefficient we obtain,

$$
K_{\text {Maxw }}(T)=\prod_{0}^{\square} \square\left(\frac{1}{2} m v^{2}\right) f_{\text {Maxw }}(v, T) d v
$$

After substituting from (A21) and (A22) we evaluate the integral (A23) and obtain,

$$
K_{\text {Maxw }}(T)=B \frac{8 \square m}{(2 \square m k T)^{3 / 2}} E_{r} e^{\frac{E_{r}}{k T}}
$$

This result must be the same as the Saha-equilibrium-based detailed-balance formula,

$$
K_{\text {Maxw }}(T)=\frac{(2 \square \hbar)^{3}}{2(2 \square m k T)^{3 / 2}} \frac{g_{z}}{g_{z+1}} A\left(L S J \square L \$ \square \square e^{\square \frac{E_{r}}{k T}}\right.
$$

where,

$$
g_{z}=2 J+1
$$

and

$$
g_{z+1}=2 J \square+1
$$

are the statistical weights of the two J-levels under consideration. The comparison between (A24) and (A25) yields the magnitude factor B, which by substituting into (A21) and (A19) gives the magnetic-sublevel electron capture cross section,

$$
\begin{gathered}
\square\left(L \$ \square M \square \square L S J M_{J}\right)=\frac{G\left(L \$ \square \square \square \square \widetilde{L} \widetilde{S} J M_{J}\right)}{G N(L \$ \square \square \square \widetilde{L} \widetilde{S} J)} \square \\
\square \frac{\square^{2} \hbar^{3}}{2 m E_{r}} \frac{g_{z}}{g_{z+1}} A\left(L S J \square L \$ \square \square \square\left(E \square E_{r}\right)\right.
\end{gathered}
$$

Finally, with (A20), (A26) and (A27), the magnetic-sublevel electron capture rate coefficient $\langle v \square\rangle$ can be evaluated, 


$$
K=f_{B}\left(E_{r}\right) \frac{G\left(L \$ \square M \square \square \widetilde{L} \widetilde{S} J M_{J}\right)}{G N(L \square \square \square \square \widetilde{L} \widetilde{S} J)} \frac{\square^{2} \hbar^{3}}{\sqrt{2 m^{3} E_{r}}} \frac{2 J+1}{2 J \square+1} A(L S J \square L \square \square \square
$$

where $f_{B}(E)$ is the electron beam distribution normalized according to,

$$
{ }_{0} f_{B}(E) d E=1
$$

\section{Acknowledgments}

This work has been supported in part by LLNL contract B503614. Work at the University of California Lawrence Livermore National Laboratory was performed under the auspices of the Department of Energy under Contract No. W-7405-Eng-48.

\section{References}

[1] I. C. Percival and M. J. Seaton, Phil.Trans. Roy. Soc. London A251, 113 (1958).

[2] E. Haug, Sol. Phys. 61, 129 (1979).

[3] E. Haug, Sol. Phys. 71, 77 (1981).

[4] M. F. Gu, D. W. Savin, and P. Beiersdorfer, J. Phys. B 32, 5371 (1999).

[5] A. Rousse, P. Audebert, J. P. Geindre, F. Falliès, J.-C. Gauthier, A. Mysyrowicz, G. Grillon, and A. Antonetti, Phys. Rev. E 50, 2200 (1994).

[6] D. D. Meyerhofer, H. Chen, J. A. Delettrez, B. Soom, S. Uchida, and B. Yaakobi, Phys. Fluids B 5, 2584 (1993).

[7] S. Bastiani, A. Rousse, J. P. Geindre, P. Audebert, C. Quoix, G. Hamoniaux,

A. Antonetti, and J.-C. Gauthier, Phys. Rev. E 56 (6), 7179 (1997).

[8] M. K. Inal and J. Dubau, J. Phys B 22, 329 (1989). 
[9] A. S. Shlyaptseva, A. M. Urnov, A. V. Vinogradov, preprint P.N. Lebedev Phys.

Inst., N. 193, 17, (Moscow, 1981).

[10] M. K. Inal and J. Dubau, J. Phys B 20, 4221 (1987).

[11] P. Beiersdorfer, D. A. Vogel, K. J. Reed, V. Decaux, J. H. Scofield, K. Widmann, G. Hölzer, E. Förster, O. Wehrhan, D. W. Savin, L. Schweikhard, Phys. Rev. A, 53 (6), 3974 (1996).

[12] S. A. Kazantsev and J.-C. Henoux, Polarization Spectroscopy of Ionized Gases, Kluwer Academic Publishers, Dorddrecht, The Netherlands (1995).

[13] T. Fujimoto, S. A. Kazantsev, Plasma Phys. Control. Fusion, 39 (9), 1267 (1997).

[14] P. Hakel, R. C. Mancini, J. C. Gauthier, E. Mínguez, J. Dubau, M. Cornille, Phys. Rev. E 69, 056405 (2004).

[15] B. W. Shore and D. H. Menzel, Principles of Atomic Spectra, Wiley (1968).

[16] E. Takács, E. S. Meyer, J. D. Gillaspy, J. R. Roberts, C. T. Chantler, L. T. Hudson, R. D. Deslattes, C. M. Brown, J. M. Laming, J. Dubau, and M. K. Inal, Phys. Rev. A, 54 (2), 1342 (1996).

[17] J. Abdallah, Jr., R. E. H. Clark, and R. D. Cowan, Los Alamos National Laboratory report LA-11436-M vol. I, (1988).

[18] R. E. H. Clark, J. Abdallah, Jr., G. Csanak, J. B. Mann, and R. D. Cowan, Los Alamos National Laboratory report LA-11436-M vol. II, (1988).

[19] R. E. H. Clark, J. B. Mann, G. Csanak, and A. L. Merts, Los Alamos National Laboratory report LA-UR-89-2675, Vol. 2, (1989). 
[20] A. S. Shlyaptseva, R. C. Mancini, P. Neill, P. Beiersdorfer, J. R. Crespo López-

Urrutia, K. Widmann, Phys. Rev. A, 57 (2), 888 (1998).

[21] A. S. Shlyaptseva, P. Hakel, and R. C. Mancini, poster paper at $11^{\text {th }}$ APS Topical Conference on Atomic Processes in Plasmas, Auburn (1998).

[22] A. S. Shlyaptseva, R. C. Mancini, P. Neill, and P. Beiersdorfer, J. Phys. B 32 (4), 1041 (1999).

[23] M. K. Inal and J. Dubau, Phys. Rev. A 47, 4794 (1993).

[24] C. D. Lin, W. R. Johnson, and A. Dalgarno, Phys. Rev. A 15 (1), 154 (1977).

[25] G. W. F. Drake, Phys. Rev. A 34, 2871 (1986).

[26] H. L. Zhang, D. H. Sampson, and R. E. H. Clark, Phys. Rev. A, 41 (1), 198 (1990).

[27] P. Hakel, Ph.D. dissertation, University of Nevada, Reno (2001).

[28] H. L. Zhang and D. H. Sampson, Phys. Rev. A 66, 042704 (2002).

[29] R. D. Cowan, The Theory of Atomic Spectra, University of California Press (Berkeley, 1981).

[30] R. Ali, C. P. Bhalla, C. L. Coke, M. Schulz and M. Stockli, Phys. Rev. A 44, 223 (1991). 
TABLE I: Relative multipole intensities of electric (E1) and magnetic (M1) dipole transitions. $\square$ and $\square$ are the polar and azimuth angles of spherical coordinates, respectively. For $\square=90^{\circ}, \hat{\square}$ defines the parallel and $\square$ the perpendicular polarization states.

\begin{tabular}{|c|c|c|c|c|}
\cline { 2 - 5 } \multicolumn{1}{c|}{} & \multicolumn{2}{c|}{ E1 } & \multicolumn{2}{c|}{$\mathrm{M} 1$} \\
\cline { 2 - 5 } \multicolumn{1}{c|}{} & $M I_{\square}$ & $M I_{\square}$ & $M I_{\square}$ & $M I_{\square}$ \\
\hline$\square \mathrm{M}=0$ & $3 \sin ^{2} \square$ & 0 & 0 & $3 \sin ^{2} \square$ \\
\hline$\square \mathrm{M}=+/-$ & $(3 / 2) \cos ^{2} \square$ & $3 / 2$ & $3 / 2$ & $(3 / 2) \cos ^{2} \square$ \\
1 & & & & \\
\hline
\end{tabular}

TABLE II: Same as in Table I but for electric (E2) and magnetic (M2) quadrupole transitions.

\begin{tabular}{|c|c|c|c|c|}
\cline { 2 - 5 } \multicolumn{1}{c|}{} & \multicolumn{2}{c|}{$\mathrm{E} 2$} & \multicolumn{2}{c|}{$\mathrm{M} 2$} \\
\cline { 2 - 5 } \multicolumn{1}{c|}{} & $M I_{\square}$ & $M I_{\square}$ & $M I_{\square}$ & $M I_{\square}$ \\
\hline$\square \mathrm{M}=0$ & $15 \sin ^{2} \square \cos ^{2} \square$ & 0 & 0 & $15 \sin ^{2} \square \cos ^{2} \square$ \\
\hline$\square \mathrm{M}=+/-$ & $(5 / 2)\left(2 \cos ^{2} \square-1\right)^{2}$ & $(5 / 2) \cos ^{2} \square$ & $(5 / 2) \cos ^{2} \square$ & $(5 / 2)\left(2 \cos ^{2} \square-1\right)^{2}$ \\
1 & & & & \\
\hline$\square \mathrm{M}=+/-$ & $(5 / 2) \sin ^{2} \square \cos ^{2} \square$ & $(5 / 2) \sin ^{2} \square$ & $(5 / 2) \sin ^{2} \square$ & $(5 / 2) \sin ^{2} \square \cos ^{2} \square$ \\
\hline
\end{tabular}




\begin{tabular}{|l|l|l|l|l|}
\hline 2 & & & & \\
\hline
\end{tabular}

TABLE III: Electron collisional excitation cross sections $1 \mathrm{~s}^{2} \square$ 1s $21(\mathrm{l}=\mathrm{s}, \mathrm{p})$ (values in units of $10^{-23} \mathrm{~cm}^{2}$ )

\begin{tabular}{|c|c|c|c|c|c|}
\hline \multirow{2}{*}{\multicolumn{2}{|c|}{$\begin{array}{c}\text { Spectral line and its upper } \\
\text { level characteristics }\end{array}$}} & \multicolumn{2}{|c|}{$6.8 \mathrm{keV}$} & \multicolumn{2}{|c|}{$8.0 \mathrm{keV}$} \\
\hline & & \multirow{2}{*}{$\begin{array}{l}\mathrm{ACE} \\
1.688\end{array}$} & \multirow{2}{*}{$\begin{array}{r}\text { Zhang } \\
2.088\end{array}$} & \multirow{2}{*}{$\begin{array}{l}\mathrm{ACE} \\
1.255\end{array}$} & \multirow{2}{*}{$\begin{array}{r}\text { Zhang } \\
1.496\end{array}$} \\
\hline$z$ & $\mathrm{M}=0$ & & & & \\
\hline $1 \mathrm{~s} 2 \mathrm{~s}^{3} \mathrm{~S}_{1}$ & $\mathrm{M}= \pm 1$ & 1.688 & 2.067 & 1.255 & 1.480 \\
\hline \multirow{2}{*}{$\begin{array}{c}y \\
1 \mathrm{~s} 2 \mathrm{p}{ }^{3} \mathrm{P}_{1}\end{array}$} & $\mathrm{M}=0$ & 3.617 & 3.644 & 3.405 & 3.379 \\
\hline & $\mathrm{M}= \pm 1$ & 5.112 & 5.261 & 3.659 & 3.784 \\
\hline \multirow{3}{*}{$\begin{array}{c}x \\
1 \mathrm{~s} 2 \mathrm{p}^{3} \mathrm{P}_{2}\end{array}$} & $\mathrm{M}=0$ & 6.206 & 6.113 & 4.155 & 4.074 \\
\hline & $\mathrm{M}= \pm 1$ & 5.026 & 4.793 & 3.360 & 3.175 \\
\hline & $\mathrm{M}= \pm 2$ & 1.484 & 1.528 & 0.974 & 0.994 \\
\hline \multirow{2}{*}{$\begin{array}{c}w \\
1 \mathrm{~s} 2 \mathrm{p}{ }^{1} \mathrm{P}_{1}\end{array}$} & $\mathrm{M}=0$ & 25.59 & 24.22 & 28.54 & 26.85 \\
\hline & $\mathrm{M}= \pm 1$ & 5.982 & 6.204 & 6.732 & 6.876 \\
\hline
\end{tabular}

TABLE IV: Polarization degrees of He-like Fe resonance lines for an electron beam energy of $6.8 \mathrm{keV}$ 


\begin{tabular}{|c|c|c|c|c|c|}
\hline & \multirow{2}{*}{ Experiment } & \multicolumn{2}{|c|}{ ACE [16] } & \multicolumn{2}{c|}{ Zhang [24] } \\
\cline { 3 - 6 } & \multirow{2}{*}{ EBIT) [11] } & 2 -level & 17 -level & 2-level & 17-level \\
\hline$w(\mathrm{E} 1)$ & $+0.56_{\square 0.08}^{+0.17}$ & +0.62 & +0.62 & +0.59 & +0.59 \\
\hline$x(\mathrm{M} 2)$ & $-0.53_{\square 0.02}^{+0.05}$ & -0.54 & -0.54 & -0.52 & -0.52 \\
\hline$y(\mathrm{E} 1)$ & $-0.22_{\square 0.02}^{+0.05}$ & -0.17 & -0.17 & -0.18 & -0.18 \\
\hline$z(\mathrm{M} 1)$ & $-0.076_{\square 0.007}^{+0.007}$ & 0 & -0.087 & -0.0051 & -0.080 \\
\hline
\end{tabular}

TABLE V: Polarization degrees of He-like Fe resonance lines for an electron beam energy of $8.0 \mathrm{keV}$

\begin{tabular}{|c|c|c|c|c|c|}
\hline \multirow{2}{*}{} & \multirow{2}{*}{$\begin{array}{c}\text { Experiment } \\
\text { (EBIT) }\end{array}$} & \multicolumn{2}{|c|}{ ACE [16] } & \multicolumn{2}{c|}{ Zhang [24] } \\
\cline { 3 - 6 } & & 2-level & 17-level & 2-level & 17-level \\
\hline$w(\mathrm{E} 1)$ & $+0.50 \pm 0.10$ & +0.62 & +0.59 & +0.59 & +0.57 \\
\hline$x(\mathrm{M} 2)$ & $-0.36 \pm 0.05$ & -0.55 & -0.53 & -0.52 & -0.49 \\
\hline$y(\mathrm{E} 1)$ & $+0.02 \pm 0.06$ & -0.036 & -0.024 & -0.057 & -0.042 \\
\hline $\boldsymbol{z}$ (M1) & $\mathbf{- 0 . 2 2} \pm \mathbf{0 . 0 7}$ & $\mathbf{0}$ & $\mathbf{- 0 . 1 5}$ & $\mathbf{- 0 . 0 0 5 5}$ & $\mathbf{- 0 . 1 4}$ \\
\hline
\end{tabular}

TABLE VI: Cascade effects on line $z$ for an electron beam energy of $8 \mathrm{keV}$

\begin{tabular}{|c|c|}
\hline kinetic model characteristics & polarization of line $z$ \\
\hline full (17-level) model & -0.15 \\
\hline
\end{tabular}




\begin{tabular}{|c|c|}
\hline 16 levels $\left(1 \mathrm{~s} 3 \mathrm{p}^{3} \mathrm{P}_{2}\right.$ excluded) & -0.08 \\
\hline ground and $\mathrm{n}=2$ levels only & -0.08 \\
\hline ground and $\mathrm{n}=2$ levels only, $1 \mathrm{~s} 2 \mathrm{p}^{3} \mathrm{P}_{2}$ excluded & 0 \\
\hline ground and $\mathrm{n}=2$ levels only, $1 \mathrm{~s} 2 \mathrm{p}^{3} \mathrm{P}_{0}$ excluded & -0.12 \\
\hline 16 levels $\left(1 \mathrm{~s} 2 \mathrm{p}^{3} \mathrm{P}_{0}\right.$ excluded $)$ & -0.18 \\
\hline 5 -level model & -0.16 \\
\hline
\end{tabular}




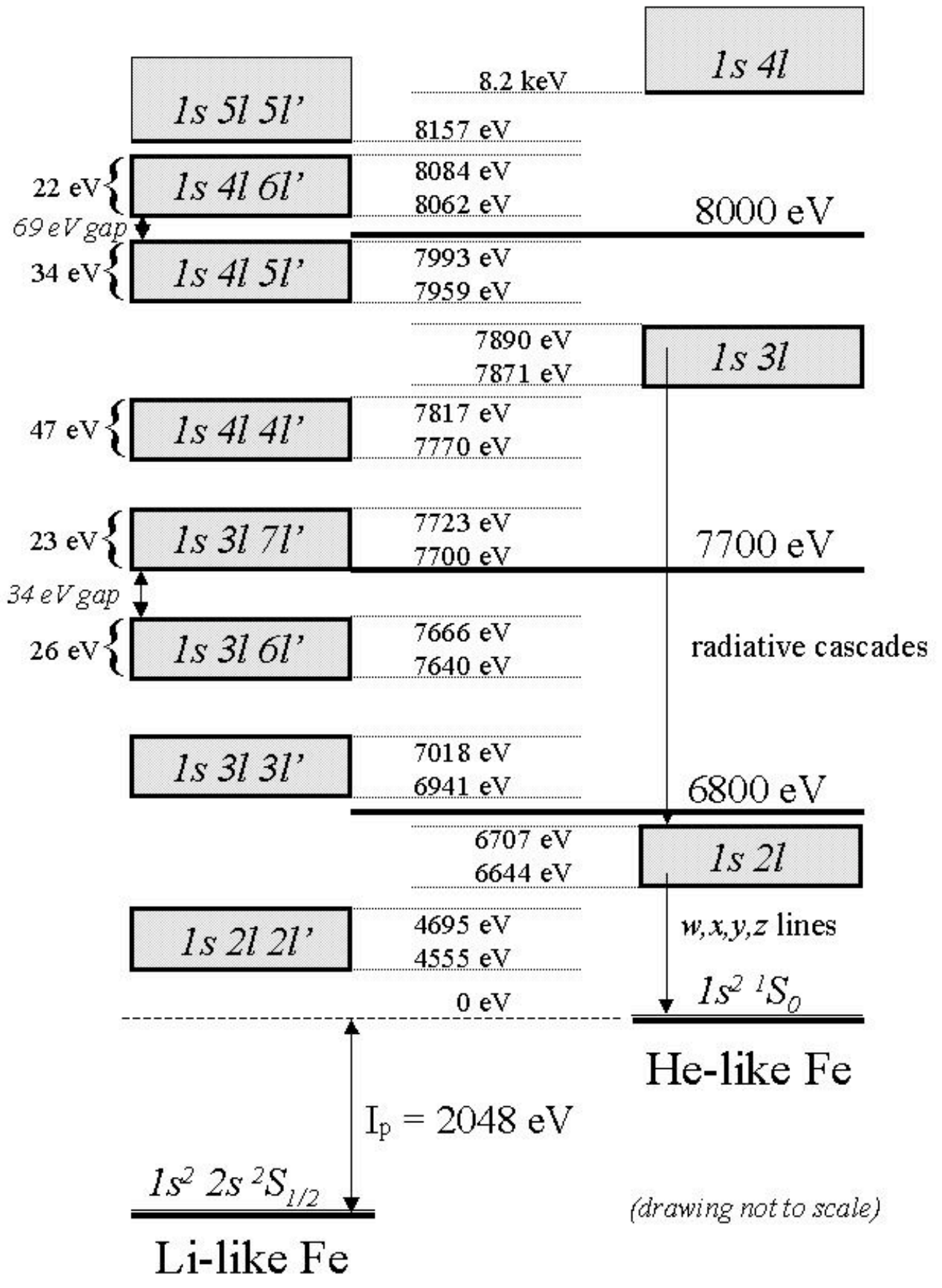

Figure 1: Energy level structure of He- and Li-like Fe. 


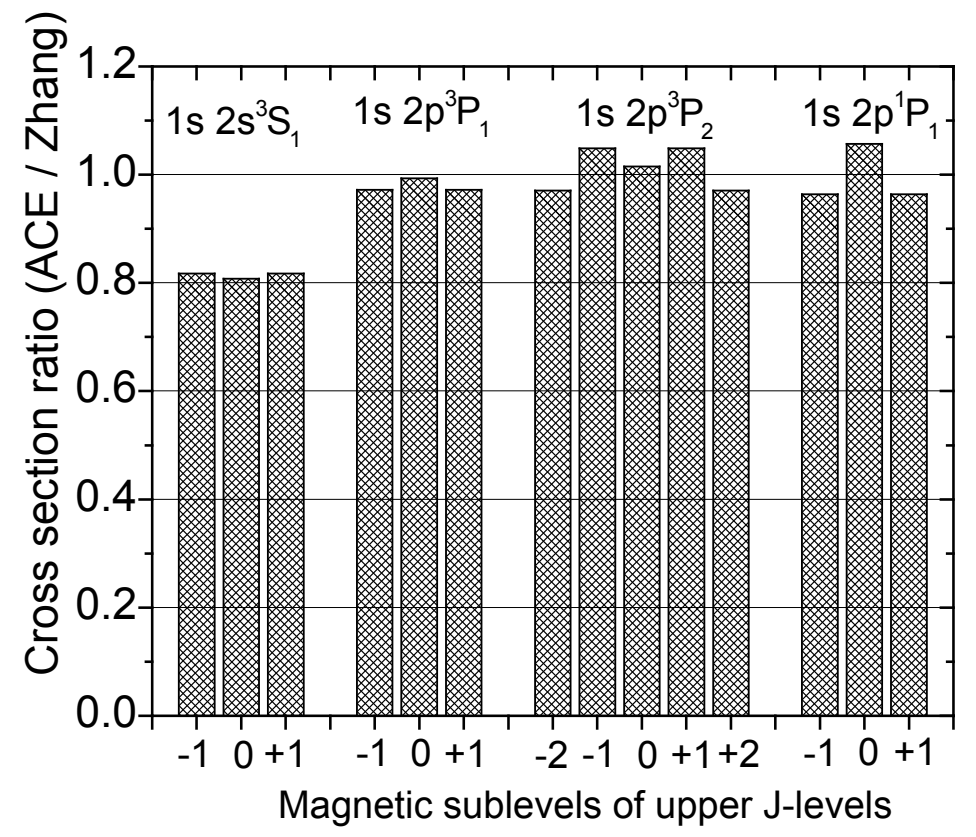

Figure 2: Comparison of magnetic sublevel cross sections from Table III for an electron beam energy of $6.8 \mathrm{keV}$.

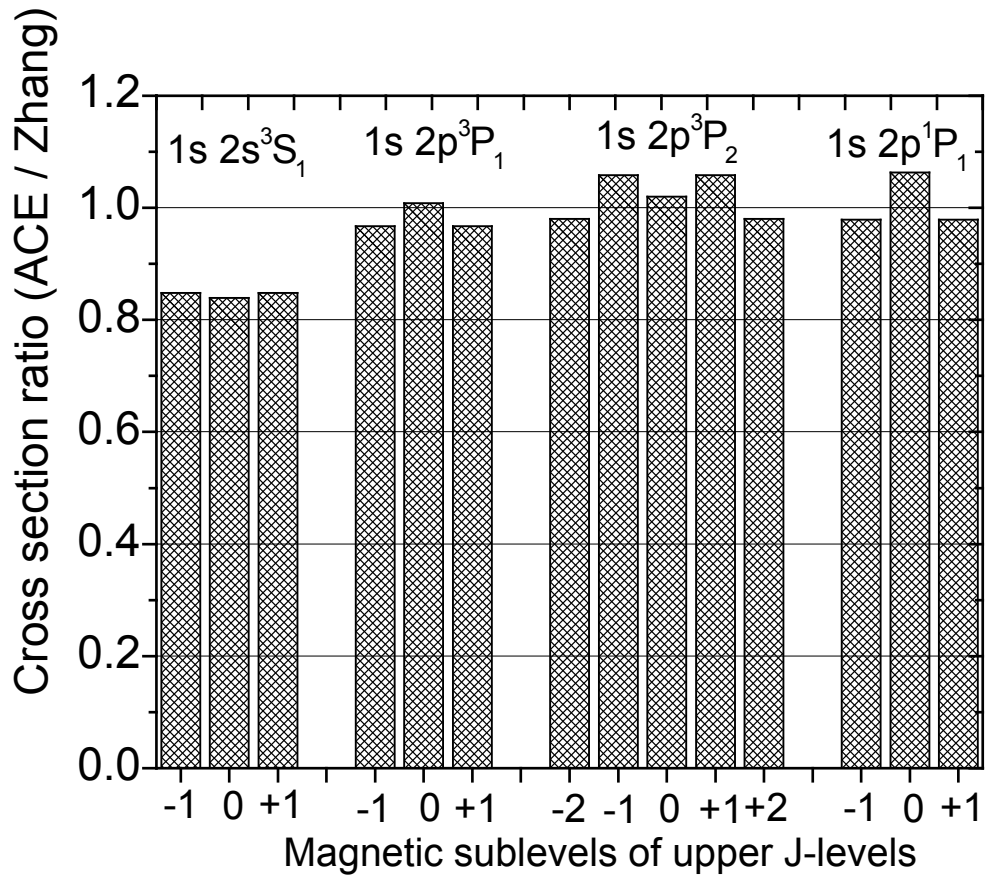

Figure 3: Same as in Fig. 2 but for an electron beam energy of $8.0 \mathrm{keV}$. 


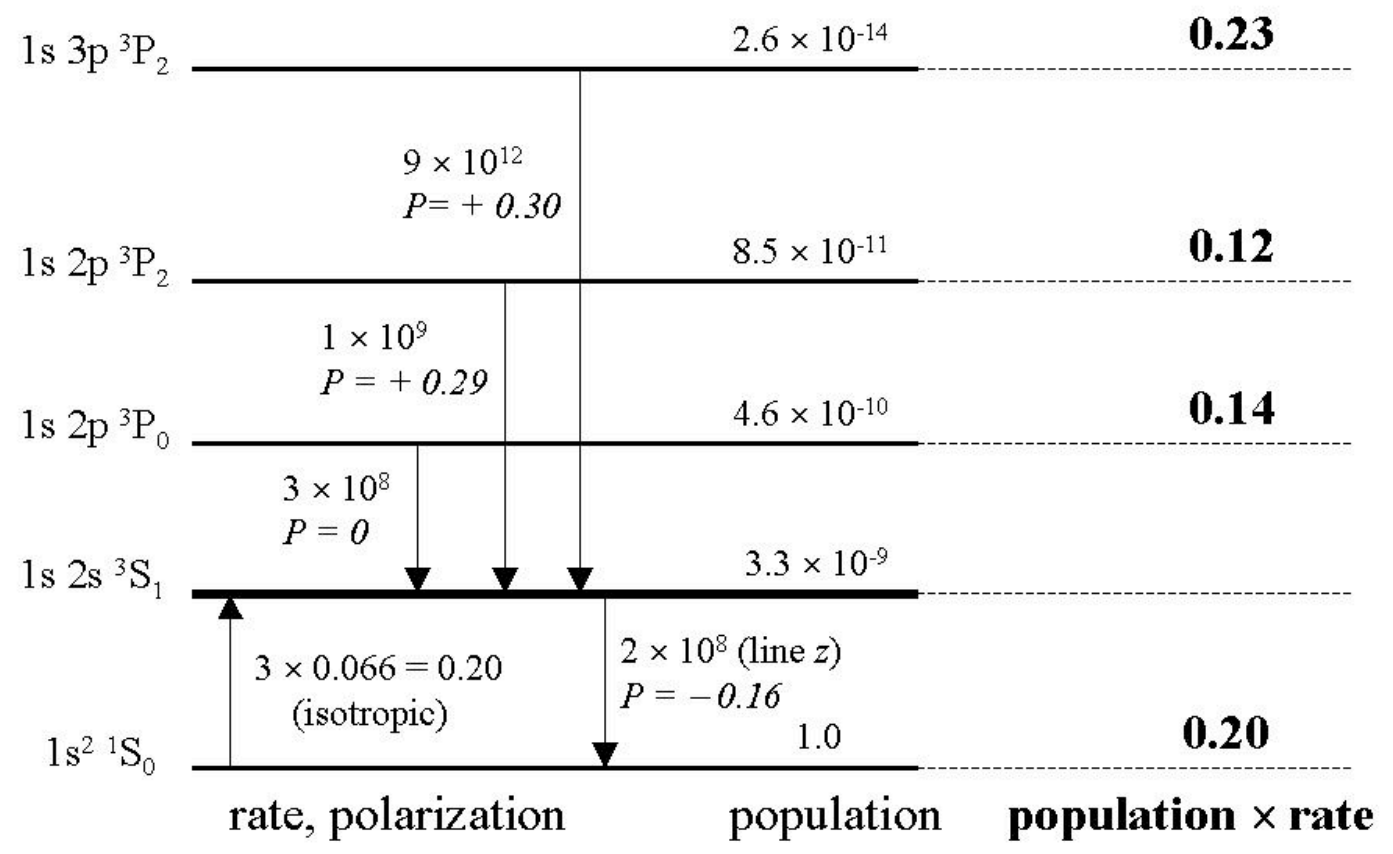

Figure 4: Dominant feeding channels for upper level of line $z\left(1 \mathrm{~s} 2 \mathrm{~s}^{3} \mathrm{~S}_{1}\right)$ for the electron beam energy of $8.0 \mathrm{keV}$. Collisional excitation and spontaneous radiative decay rates are in $\mathrm{s}^{-1}$. Level populations are normalized to the population of the ground state. 